RSCPublishing Materials Chemistry

\title{
Exploiting the Localized Surface Plasmon Modes in Gold Triangular Nanoparticles for Sensing Applications
}

\begin{tabular}{|r|l|}
\hline Journal: & Journal of Materials Chemistry \\
\hline Manuscript ID: & JM-ART-02-2012-030944 \\
\hline Article Type: & Paper \\
\hline Date Submitted by the Author: & 15 -Feb-2012 \\
\hline Complete List of Authors: & $\begin{array}{l}\text { Morarescu, Rodica; Université de Mons, Laboratoire Interfaces \& Fluides } \\
\text { Complexes, Centre d' Innovation et de Recherche en Matériaux Polymères } \\
\text { Kolaric, Branko; UNIVERSITE MONS, INFLUX lab } \\
\text { damman, pascal; University of Mons - UMONS, InFluX Laboratory, } \\
\text { Maes, Bjorn; UNIVERSITE MONS, INFLUX lab } \\
\text { Vallée, Renaud; Centre de Recherche Paul Pascal, } \\
\text { Shen, Honghui; Ghent University-IMEC, }\end{array}$ \\
\hline
\end{tabular}




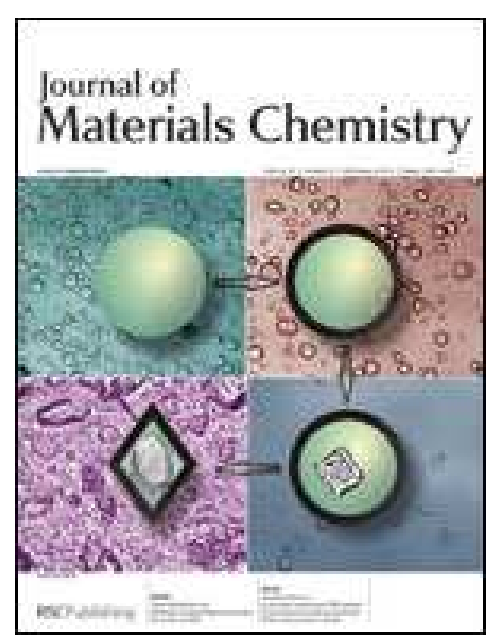

\section{Journal of Materials Chemistry} Full paper submission

Journal of Materials Chemistry is a weekly publication from the Royal Society of Chemistry, covering all aspects of materials chemistry: the fabrication, properties and applications of materials, including synthesis, structural characterisation and modelling.

2010 impact factor: 5.1

The fastest times to publication in the field www.rsc.org/materials

The following paper has been submitted to Journal of Materials Chemistry for consideration as a full paper.

Journal of Materials Chemistry wishes to publish original research that demonstrates novelty and advance, either in the chemistry used to produce materials or in the properties/applications of the materials produced. Work submitted that is outside of these criteria will not usually be considered for publication.

Routine or incremental work, however competently researched and reported, should not be recommended for publication if it does not meet our expectations with regard to novelty and impact.

It is the responsibility of authors to provide fully convincing evidence for the homogeneity and identity of all compounds they claim as new. Evidence of both purity and identity is required to establish that the properties and constants reported are those of the compound with the new structure claimed.

Thank you for your effort in reviewing this submission. It is only through the continued service of referees that we can maintain both the high quality of the publication and the rapid response times to authors. We would greatly appreciate if you could review this paper in two weeks. Please let us know if that will not be possible.

Once again, we appreciate your time in serving as a reviewer. To acknowledge this, the RSC offers a $\mathbf{2 5 \%}$ discount on its books: http://www.rsc.org/Shop/books/discounts.asp. Please also consider submitting your next manuscript to Journal of Materials Chemistry.

Best wishes,

$$
\text { liz Davies }
$$

Liz Davies

Editor, Journal of Materials Chemistry 


\section{Dr Rodica Morarescu}

Laboratoire Interfaces \& Fluides Complexes,

Centre d'Innovation et de Recherche en Matériaux Polymères,

Université de Mons Hainaut, 20 Place du Parc, 7000 Mons, Belgium

e-mail: rodica.morarescu@umons.ac.be

Mons 15.02.2012

\section{Dear Editor,}

Please find herewith our manuscript "Exploiting the Localized Surface Plasmon Modes in Gold Triangular Nanoparticles for Sensing Applications" which we submit for publication in Journal of Material Chemistry as regular article.

Our motivation was to reveal the origin of the plasmonic response in arrays of gold triangles with high aspect ratios. Experimental and theoretical investigation clearly assign the observed plasmonic peaks to the quadrupole and dipole modes. Additionally these modes have been investigated for possible sensing applications. We clearly demonstrate that dipole mode is significantly more sensitive than quadropole mode. As it is pointed in the manuscript, we have shown that the plasmon modes observed for larger triangles exhibit unexpected sensitivity with change in refractive index, attributed to an increase of the local field enhancement for sharper tips.

We think that the novelty of this study from physical as well as from material perspective justify the submission of our manuscript to Journal of Material Chemistry and we hope that you will find it appropriate to merit publication.

Please note that this manuscript has never been copyrighted, classified or published before, nor is it being considered for publication elsewhere. Also, all authors agree with the contents of the version.

In the case of any additional questions, please do not hesitate to contact me.

Most sincerely yours,

\section{Rodica Morarescu}




\title{
Exploiting the Localized Surface Plasmon Modes in Gold Triangular Nanoparticles for Sensing Applications
}

\author{
Rodica Morarescu ${ }^{1}{ }^{*}$ Honghui Shen ${ }^{2}$, Renaud A. L. Vallée ${ }^{3}$, \\ Bjorn Maes ${ }^{2,4}$, Branko Kolaric ${ }^{1}$, and Pascal Damman ${ }^{1}$ \\ ${ }^{1}$ Laboratoire Interfaces $\&$ Fluides Complexes, \\ Centre d'Innovation et de Recherche en Matériaux Polymères, \\ Université de Mons, 20 Place du Parc, B-7000 Mons, Belgium \\ ${ }^{2}$ Photonics Research Group (INTEC), Ghent University-Imec, \\ Sint-Pietersnieuwstraat 41, B-9000 Ghent, Belgium \\ ${ }^{3}$ Centre de Recherche Paul Pascal (CNRS-UPR8641), \\ 115 avenue du docteur Schweitzer, 33600 Pessac, France and \\ ${ }^{4}$ Micro- and Nanophotonic Materials Group, \\ Faculty of Science, University of Mons, \\ 20 Place du Parc, B-7000 Mons, Belgium
}

\begin{abstract}
In this study we investigate and exploit, for optical sensing, the surface plasmon excitation in gold triangular nanoparticles with high aspect ratios (i.e., the ratio of edge length of the triangles with the height) prepared by nanosphere lithography. As shown previously, shape and size of these nanoparticles were used to tune their optical properties, monitored by far field extinction spectroscopy. Interestingly, several localized surface plasmon resonances were detected in the visible and near infrared regions and attributed to dipole and quadrupole modes. These modes, identified from numerical simulations, "red shift" as the aspect ratio of the particles increases. The plasmon modes observed for larger triangles exhibit unexpected sensitivity with change in the refractive index. From experiments and numerical simulations, this higher sensitivity has been attributed to an increase of the local field enhancement for sharper tips. This new effect can be an important information for the design of particles as building blocks for sensing applications.
\end{abstract}

*Electronic address: rodica.morarescu@umons.ac.be 


\section{INTRODUCTION}

Plasmonic nanostructures have received increasing attention in recent decades due to their unique optical properties. These properties are dominated by localized surface plasmon polariton resonances (LSPPRs), i.e. collective oscillations of the conduction band electrons, driven by an incident electromagnetic field. The frequency, the width, and the amplitude of these resonances strongly depend on the morphology of the nanoparticles (NPs), in particular on their size and shape, on the dielectric function of the surrounding medium. The collective oscillation of the conduction band electrons is accompanied by an enhancement of the local field in the vicinity of the NP surface. Various chemical and biological applications take advantage of the high sensitivity of LSPPRs to the refractive index of the medium in the vicinity of the metal surface. Small changes in the local refractive index yield spectral shifts of the LSPPR peak observed in the extinction spectra. This shift in wavelength per unit change in the refractive index (RIU) of the surrounding medium is used to quantify the sensitivity factor $S$ of the NPs.

There is a permanent interest to develop and to improve the sensitivity of optical sensors based on metal NPs $[1,2]$. A variety of chemical synthesis and lithographic techniques have opened possibilities to design new plasmonic structures with defined size and shape, dispersed in solutions or supported on substrates. In particular, arrays of supported monodisperse NPs represent potential structures for many applications such as optical sensing [3], surface enhanced Raman scattering (SERS) [4-6], surface-enhanced fluorescence microscopy [7], nanopatterning $[8,9]$ and solar cells $[10,11]$. Conventional lithography techniques, such as electron beam lithography (EBL) [12, 13] and focused ion beam lithography (FIB) [14], can routinely fabricate ordered arrays of metal NPs. Besides these standard techniques, new methods taking advantage of the self-assembly of colloids (nanosphere lithography, NSL) [15] were developed to produce regular arrays of gold metal NPs. The main advantage of this powerful technique is low-cost fabrication of large arrays of NPs with controlled size, shape and interparticle distance. NP arrays prepared by NSL have been extensively studied last years. For instance, Jensen et al demonstrated the ability of NSL to tune the optical properties of triangular NPs across the visible and near IR region [16].

Although many studies were reported, a detailed investigation of triangular NPs with very high aspect ratios (AR, i.e. ratio of edge length and height) is still missing. The present 
work is aimed to investigate the optical properties of such gold triangular NPs prepared by NSL with AR ranging between 2.7 and 7.2. The extinction spectra of these NP arrays reveal the presence of two intense plasmon resonances that were attributed to dipole and quadrupole modes spectrally resolved in the visible and IR region. In addition, increasing the AR produces a gradual red-shift of the LSPP resonances that drastically improves the spectral resolution of the modes. Finally we demonstrate that these larger NPs are superior candidates for (bio)sensing applications thanks to their very high sensitivity to change in the refractive index of the surrounding medium. This higher sensitivity was associated to the stronger local field enhancement reached in the vicinity of the sharp tips of the larger NPs.

\section{EXPERIMENTAL TECHNIQUES}

As mentioned above, experiments presented here use arrays of triangular gold NPs prepared by NSL [18], utilizing the drop coating method of Micheletto et al. [19]. The technique employs nanospheres dispersed in solution to create a close-packed monolayer with hexagonal symmetry, used as a mask in a subsequent step, when gold is deposited through the nanospheres to fill the void spaces among the layer lattice. In this way regular arrays of triangular gold NPs are fabricated in a last step, when the nanosphere mask is removed. NSL has the advantage of making it possible to largely tune the AR of the NPs by changing the size of the nanospheres used as a mask and/or by changing the thickness of the evaporated gold. In this contribution monolayers of nanospheres with a diameter $D$ of $356 \mathrm{~nm}, 456 \mathrm{~nm}$, $628 \mathrm{~nm}$ and $771 \mathrm{~nm}$ have been prepared on fused silica substrates and used as a lithographic mask in a subsequent step when gold was evaporated. The gold film thickness determined by a quartz crystal microbalance has been fixed to $40 \mathrm{~nm}$. After the nanosphere masks are removed triangular NP arrays with the following edge length remain on the substrates: $l=$ $109 \mathrm{~nm}, 135 \mathrm{~nm}, 180 \mathrm{~nm}$ and $288 \mathrm{~nm}$. The Aspect Ratio of these NPs increases linearly with the edge length as following 2.7 (array $\mathrm{AR}_{2.7}$ ), 3.4 (array $\left.\mathrm{AR}_{3.4}\right), 4.5\left(\right.$ array $\mathrm{AR}_{4.5}$ ) and $7.2\left(\right.$ array $\left.\mathrm{AR}_{7.2}\right)$.

The AR of the NPs can also be tuned by changing the thickness of the evaporated gold for a constant nanosphere diameter. In this context, for the NPs designed using nanospheres with diameter $D=771 \mathrm{~nm}$, in a second step $80 \mathrm{~nm}$ gold was evaporated instead of $40 \mathrm{~nm}$. 
As a consequence, the AR of the NPs has been changed from 7.2 (array $\mathrm{AR}_{7.2}$ ) to 3.6 (array $\left.\mathrm{AR}_{3.6}^{*}\right)$.

The samples have been characterized by atomic force microscopy and the edge length values of the NPs presented above have not been deconvoluted for tip broadening effects. In order to monitor the optical properties of triangular NPs, far field visible-NIR extinction spectroscopy in the wavelength range 400 - $1800 \mathrm{~nm}$ has been applied. All the extinction spectra here presented are recorded using unpolarized light, under normal incidence with respect to the sample surface. In order to probe the sensitivity of the NPs the extinction spectra were recorded when the samples were placed in different environments with a different refractive index.

\section{RESULTS AND DISCUSSION}

\section{A. Investigation of Multipolar Plasmon Modes}

Figure 1 shows the AFM images of different highly ordered triangular NPs used in this study. The experimental extinction spectra of these arrays are presented in figure $2 \mathrm{~A}$.
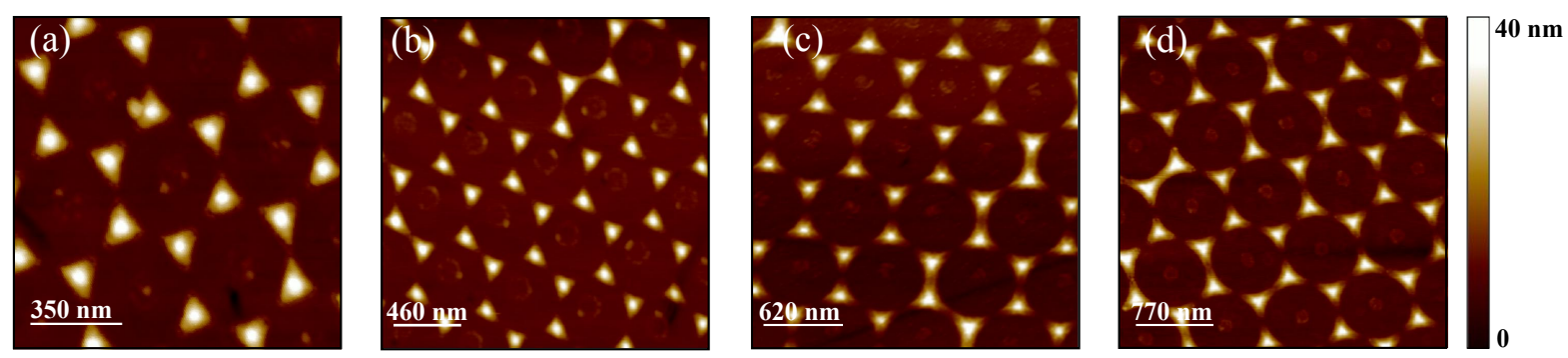

FIG. 1: AFM images of triangular NP arrays with various ARs, (a) $\mathrm{AR}_{2.7}$, (b) $\mathrm{AR}_{3.4}$, (c) $\mathrm{AR}_{4.5}$ and (d) $\mathrm{AR}_{7.2}$. See text for details about these arrays.

For array $\mathrm{AR}_{2.7}$ the dominant resonance is an extinction band at $851 \mathrm{~nm}$. Additionally a weaker band arises at $609 \mathrm{~nm}$. Increasing the AR at constant gold thickness leads to a gradual red shift of these resonances from the array $\mathrm{AR}_{2.7}$ to the array $\mathrm{AR}_{7.2}$. For example for array $\mathrm{AR}_{7.2}$ the strong and weak bands are located at $1463 \mathrm{~nm}$ and $730 \mathrm{~nm}$, respectively. As the AR of the triangles increases, we also observe an increase of their intensity, bandwidth and a better separation of both LSPP resonances. 

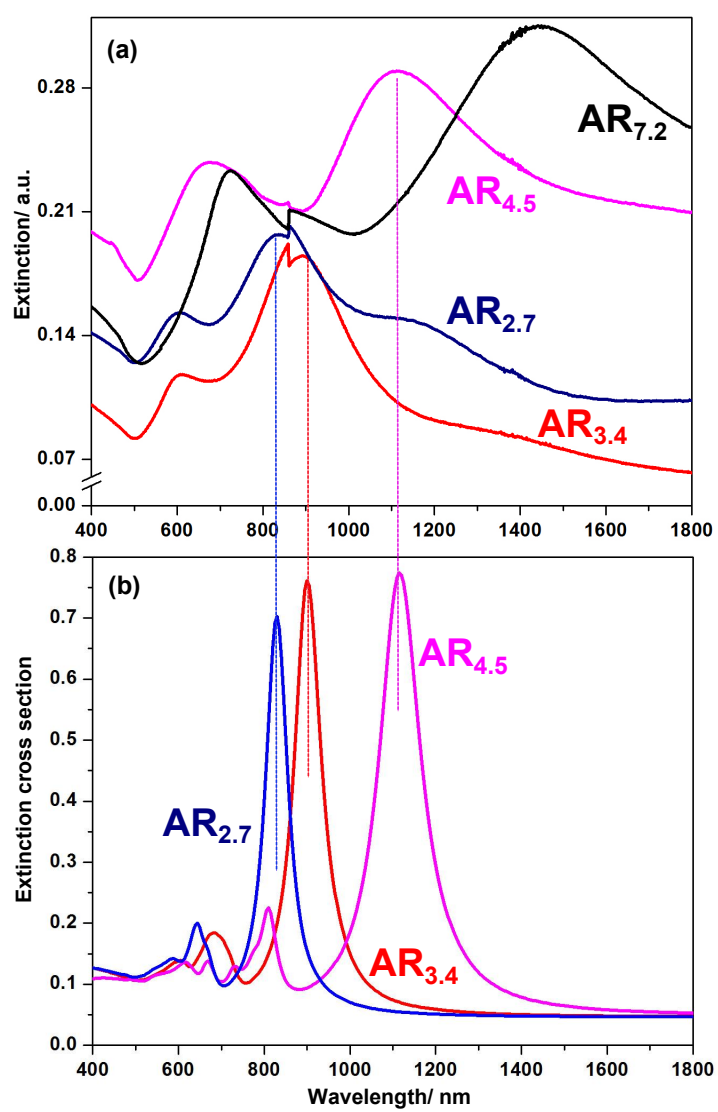

FIG. 2: (a) Experimental extinction spectra of NP arrays with different aspect ratios $\mathrm{AR}_{2.7}, \mathrm{AR}_{3.4}$, $\mathrm{AR}_{4.5}$ and $\mathrm{AR}_{7.2}$, as indicated. The spectra were recorded under normal incidence using unpolarized light. (b) Simulated extinction spectra of triangular NPs array $\mathrm{AR}_{2.7}, \mathrm{AR}_{3.4}$ and $\mathrm{AR}_{4.5}$.

Changing the gold thickness also affects the optical properties of the NPs array. Figure 3 shows that the dipole resonance experienced a $440 \mathrm{~nm}$ blue shift from array $\mathrm{AR}_{7.2}$ to array $\mathrm{AR}_{3.6}^{*}$ (same edge length but different height, 40 and $80 \mathrm{~nm}$, respectively). Additionally a narrowing of plasmon width has been observed due to the decrease of the AR.

To have a better insight into the optical properties of these triangular NPs we simulate the extinction spectra of the NPs arrays by finite-element COMSOL simulations. Figure 4 depicts the schematic representation of the triangular NP geometry used in the simulations: (a) perspective view and (b) top view. The polarization direction has been chosen along the bisector of the triangular NPs. Only the in plane excitation has been investigated. The sharpness of the tips has been controlled by adjusting the angle $\theta(\theta=2 L / D$ is a geometrical 


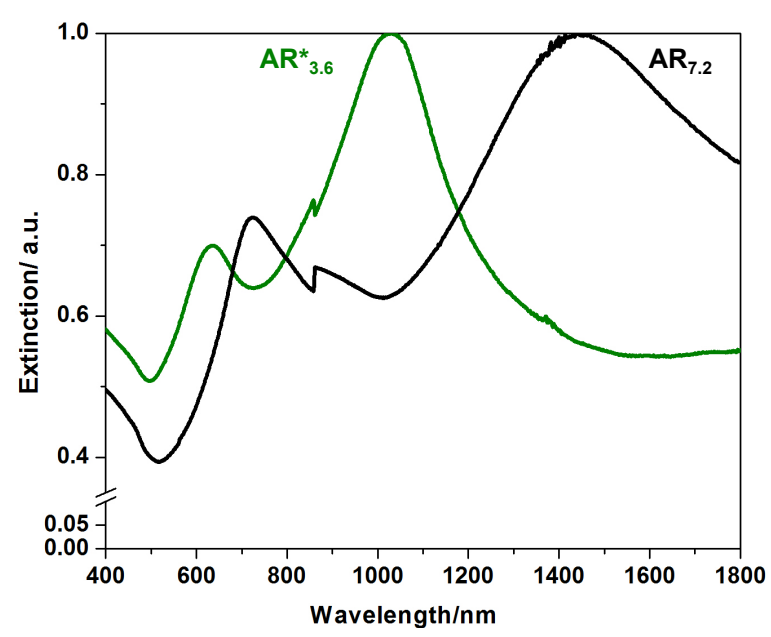

FIG. 3: Experimental extinction spectra of NPs array prepared using the same diameter of the nanospheres $D=771 \mathrm{~nm}$ but different gold thickness: $40 \mathrm{~nm}\left(\mathrm{AR}_{7.2}\right)$ and $80 \mathrm{~nm}\left(\mathrm{AR}_{3.6}^{*}\right)$.

parameter without dimension describing the sharpness of the tip, see Fig. 4). A decrease of $\theta$ is obviously related to an increase of tip sharpness. Please note that care has to be given to the geometric parameters used to model the experiments, in order to fall well within the range of experimental measured values. For each specific NPs array geometry, the $\theta$ angle was adjusted to have a good agreement with the experimental extinction spectra. For the NPs array $\mathrm{AR}_{2.7}, \mathrm{AR}_{3.4}$ and $\mathrm{AR}_{4.5}$, we used $\theta$ values of $0.49\left(28^{\circ}\right), 0.49\left(28^{\circ}\right)$ and $0.42(24$ $\left.{ }^{\circ}\right)$, respectively. The largest $\mathrm{NPs}\left(\mathrm{AR}_{4.5}\right.$, edge length $\left.180 \mathrm{~nm}\right)$ have thus the sharpest tips (smallest $\theta$ ).

The simulated extinction spectra of triangular $\mathrm{NP}$ arrays $\mathrm{AR}_{2.7}, \mathrm{AR}_{3.4}$ and $\mathrm{AR}_{4.5}$ are given in figure 2B. Similar trends as in the experimental spectra are observed: i) the presence of two significant resonances, ii) these resonances are gradually red-shifted and iii) their bandwidth increases with increasing edge length of the triangles. Additionally for array $\mathrm{AR}_{4.5}$, besides the two main resonances, multiple weak higher-order peaks show up around $670 \mathrm{~nm}$.

Interestingly, the experimental spectra can be adequately reproduced with a single "fitting "parameter the $\theta$ angle. For all arrays, the position of the maximum LSPPR for the fundamental resonance $(l=1)$ of the calculated spectra matches well the experimental ones within $\pm 20 \mathrm{~nm}$. For the second order LSPP resonance $(l=2)$, the calculated peaks are slightly red-shifted compared to the experimental ones. Nevertheless, it should be noted that in the calculations these resonances appear slightly split into two modes. A slight blue 
shift can be observed in the experimental spectra due to a possible overlapping of the peaks and experimental broadening. We assign the fundamental sharp resonance to the dipolar mode and the second order resonance observed for shorter wavelengths to the quadrupole mode. For array $\mathrm{AR}_{4.5}$ a weak resonance is observed at $670 \mathrm{~nm}$ in the simulated spectra that may be attributed to the excitation of multipolar modes higher than $l=2$.

Figure 5 reveals the linear dependence of the experimentally determined wavelengths corresponding to the maximum extinction bands of the dipole and quadrupole modes versus the aspect ratios, as expected for such triangular NPs arrays [23].

(a)

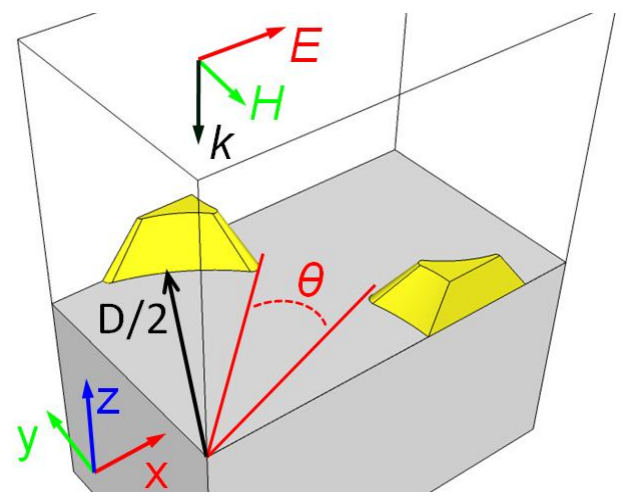

(b)

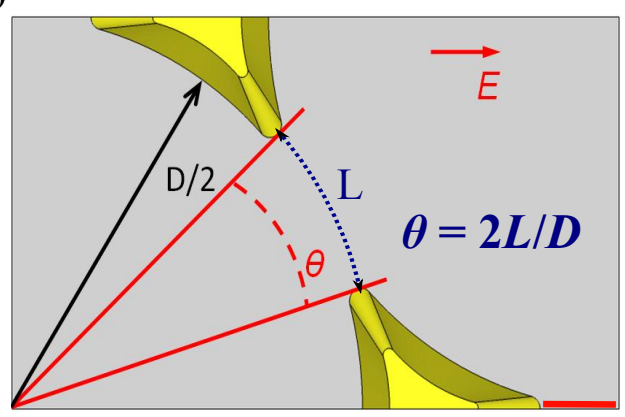

FIG. 4: Schematic representation of triangular NPs geometry as used in COMSOL simulations: (a) perspective view and (b) top view respectively. The sharpness of the tips has been controlled by adjusting the angle $(\theta)$.

Besides the far field properties, the near field optical properties of the triangular NP arrays $\mathrm{AR}_{2.7}, \mathrm{AR}_{3.4}$ and $\mathrm{AR}_{4.5}$ have been numerically investigated. Figure 6 shows the calculated electric field maps close to the particle surface of array $\mathrm{AR}_{4.5}$ for the LSPP resonances attributed to the dipole $(\lambda=1115 \mathrm{~nm}(\mathrm{a}))$ and quadrupole $(\lambda=810 \mathrm{~nm}(\mathrm{~b}))$ excitations. These electric field maps correspond to NPs arrays supported on fused silica substrate and a polarization direction along the bisector of the triangles. For the dipole and quadrupole resonances, the largest fields are generated on the NPs tips that are aligned with the polarization direction (see figure 6A and B). Negligible field enhancements can be observed for the tips perpendicular to the polarization direction.

It is well know that the optical properties of metal NPs are influenced by the particle size. 


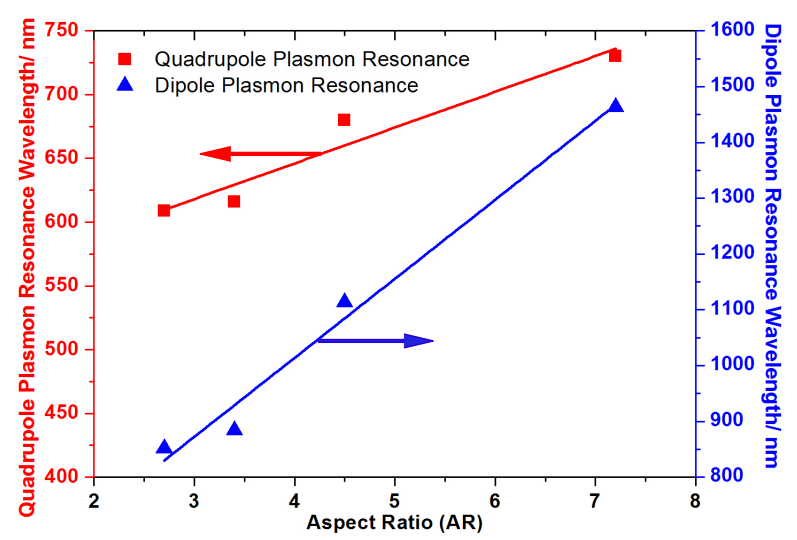

FIG. 5: Linear relationship between the experimental LSPP resonance wavelengths of the dipole (blue) and quadrupole (red) vs the aspect ratios of the gold nanotriangles. All the extinction spectra have been recorded in air. The axes are as indicated.

(a)

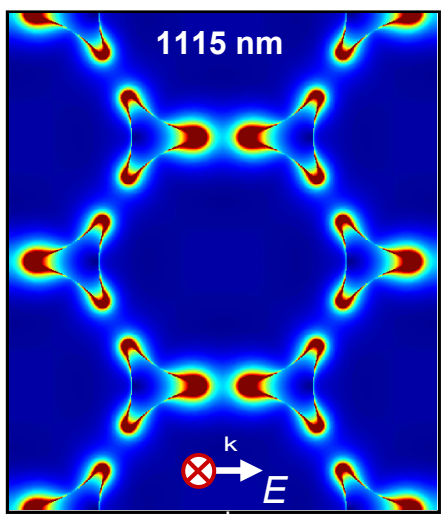

(b)

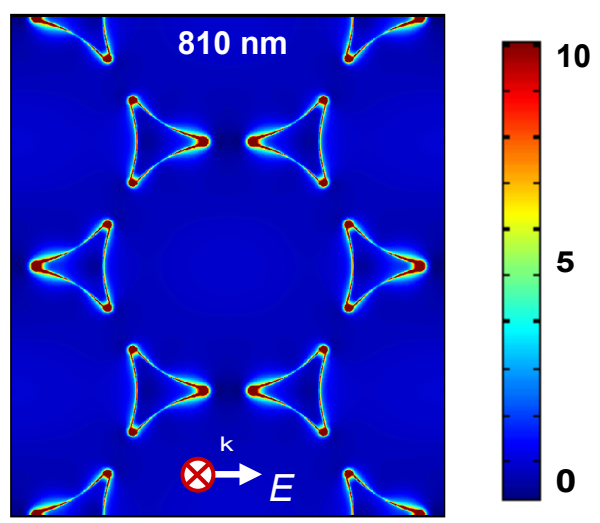

FIG. 6: 2D maps representing the square of the electric field external to triangular gold NP array $\mathrm{AR}_{4.5}$. The sharpness of the NPs has been chosen by fixing the angle $\theta$ at $24^{\circ}$. The polarization of light is along the triangles bisector. The map has been calculated for the LSPP resonances which correspond to the dipole resonance $\lambda=1115 \mathrm{~nm}$ (a) and quadrupolar resonance $\lambda=810 \mathrm{~nm}$ (b).

With increasing particle size higher order plasmon modes appear in the extinction spectrum [21]. These results can be explained by considering that for larger NPs, the incoming light can not polarize the particle homogeneously. As a consequence, retardation effects lead to a gradient of the electromagnetic field, that excite higher order modes. A broadening of the plasmon bandwidth is observed for large NPs as a result of radiative damping. The retardation effects within the particles lead to considerable decrease of the plasmon lifetime 
and thus the dipolar near field enhancement.

The optical properties of metal NPs are also strongly influenced by the particle shape. This effect is obvious for very different geometries such as spheres, rods, squares and triangles. In a more subtle way, it has been shown recently by Sherry et al. that the sharpness of the tips for triangular NPs affects drastically their optical properties[17]. The drastic influence of the snipping effect (i.e. rounding of the tips) on optical properties of triangular NPs has been concomitantly reported [22].

For the design of NPs and the tuning of their optical properties, both size and shape are thus of a crucial importance. For NPs arrays prepared by NSL, the sharpness of the tips is clearly affected by a change of the colloidal spheres diameter (see Figs. 1 and 2). The influence of the tip sharpness (determined by the angle $\theta$ ) was investigated with COMSOL simulations.
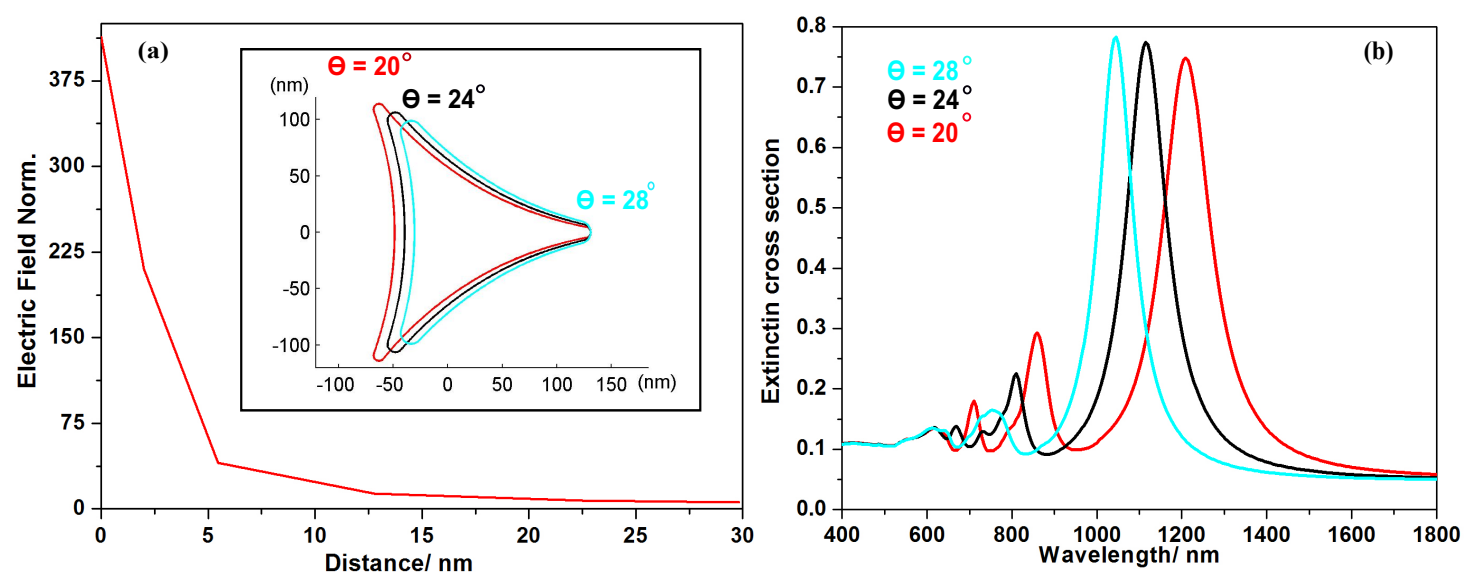

FIG. 7: (a) Evolution of normalized electric field with distance from the tip for a triangular NPs array $\mathrm{AR}_{4.5}$ and a angle $\theta$ equal to $20^{\circ}$. The electric field corresponds to the dipolar mode. The inset shows the shape of the triangles with different sharpness used in the simulations $\theta: 20^{\circ}, 24^{\circ}$, $28^{\circ}$. (b) Evolution of the extinction spectra of similar triangular NPs with various $\theta$ angle, as indicated.

Figure 7a depicts the rapid decrease of the electric field with the distance from the tip, for the dipole mode of NPs array $\mathrm{AR}_{4.5}$. From the extinction spectra of these NPs (Figure $7 \mathrm{~b}$ ), we see that the dipole resonance is very sensitive to the tip sharpness, red shifts as large as $170 \mathrm{~nm}$ are observed for the sharpest triangles $\left(\theta=20^{\circ}\right)$. 
In order to understand why a change in tip sharpness produces such dramatic effect on the optical spectra of the NPs, some authors consider that the sharp tips on a perfect triangle lead to polarization distortions that favor higher order multipoles [23]. For snipped triangular NPs, this effect is reduced and the electric field becomes more homogeneous.

\section{B. Optical Sensing based on Exploitation of Plasmon Modes in Triangular NPs}

As shown in Fig. 8, the size of the triangular NPs affect the field enhancement near the tip. From COMSOL simulations, we can see that the field decays away from the tip surface faster for smaller NPs. If we consider that higher enhancement of the near fields correlates with a higher sensitivity, then we can conclude that the largest NPs with sharper tips are excellent candidates for sensing applications.

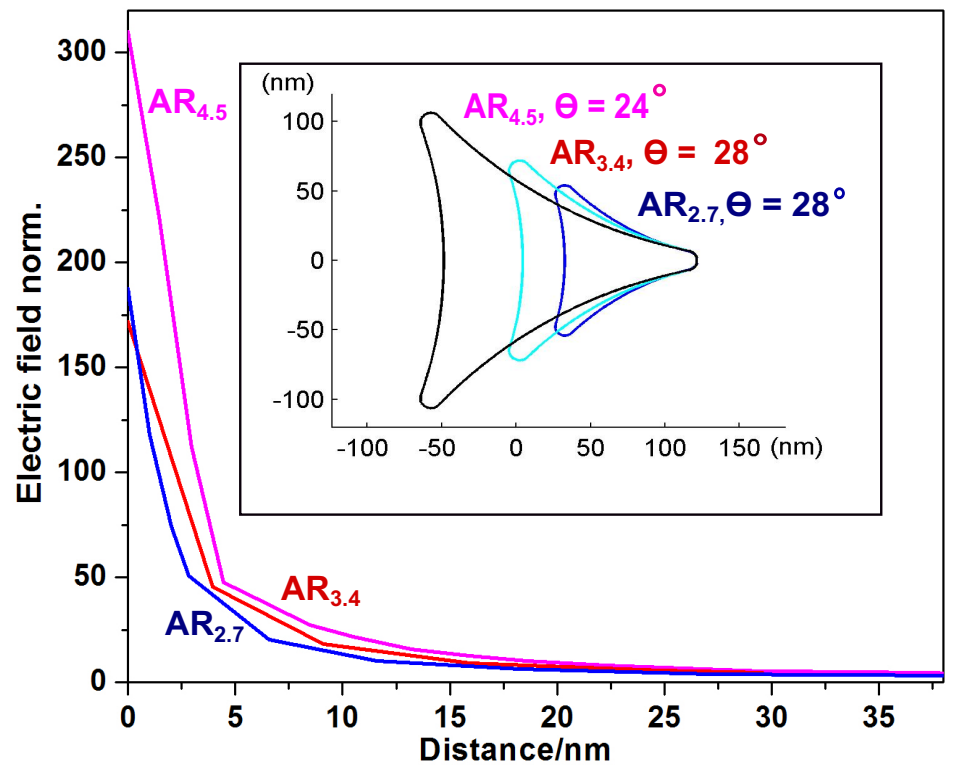

FIG. 8: Electric field normalized (dipole excitation) for NPs arrays $\mathrm{AR}_{2.7}, \mathrm{AR}_{3.4}$ and $\mathrm{AR}_{4.5}$. The sharpness of the tips was adjusted to reproduce experimental spectra. The inset (a) shows the shape of the triangles with different sharpness used in the COMSOL simulations.

In the following, we demonstrate experimentally that the largest NPs are the most sensitive. Shifts of the LSPP peak position for dipole and quadrupole resonances are observed by changing the refractive index of surrounding solvent (Fig. 9). This give us the opportunity 
to measure the sensitivity (given by wavelength shift per RIU) for the NPs arrays $\mathrm{AR}_{2.7}$, $\mathrm{AR}_{3.4}$ and $\mathrm{AR}_{4.5}$.
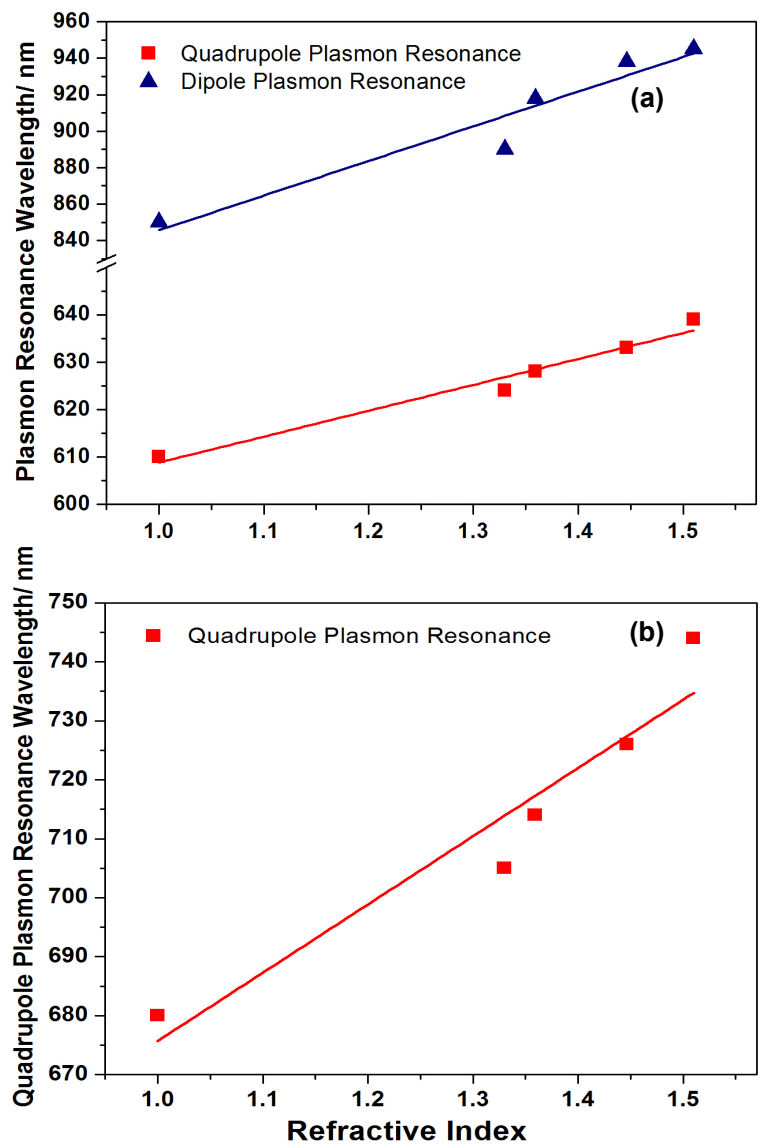

FIG. 9: (a) Linear relationship between the experimental LSPP resonance wavelengths of the dipole (blue) and quadrupole (red) of the NPs array $\mathrm{AR}_{2.7}$ vs the refractive index of different solvents. (b) Linear relationship between the experimental LSPP resonance wavelengths of the quadrupole (red) of the NPs array $\mathrm{AR}_{4.5}$ vs the surrounding refractive index.

Figure 9A shows the evolution of LSPPR peak position (dipole and quadrupole) for NPs array $\mathrm{AR}_{2.7}$ with the surrounding refractive index. From these data, sensitivity factors of $190 \mathrm{~nm} / \mathrm{RIU}$ for dipole and $54 \mathrm{~nm} / \mathrm{RIU}$ for the quadrupole are obtained. For the NPs array $\mathrm{AR}_{4.5}$, only the sensitivity of the quadrupole resonance $(116 \mathrm{~nm} / \mathrm{RIU}$ ) was determined (see Figure 9B). Due to the near - IR absorption of the the solvents used for tuning the refractive index, it was not possible to record the LSPPR shift of the dipole mode.

However, the shift observed for the dipole resonance of the array of largest particles $\mathrm{AR}_{4.5}$ 
immersed in pyridine is two times larger than those observed for array of smallest particles $\mathrm{AR}_{2.7}$ immersed in the same solvent.

For the studied NPs arrays, the dipole resonance is more sensitive to change in refractive index compared to quadrupole resonance. The sensitivity of the triangular NPs clearly increases with increasing their edge length at constant gold thickness.

To further support this observation and avoid the near-IR absorption of solvent, the sensitivity of large particles, $D=771 \mathrm{~nm}$ with a gold thickness of $80 \mathrm{~nm}$ (array $\mathrm{AR}_{3.6}^{*}$ ) has been investigated. The resulting sensitivity will be a lower bound of the value expected for the $40 \mathrm{~nm}$ array $\mathrm{AR}_{7.2}$. Indeed, Hayes et al. reported that the triangular NPs with the smallest thickness are the most sensitive [24].

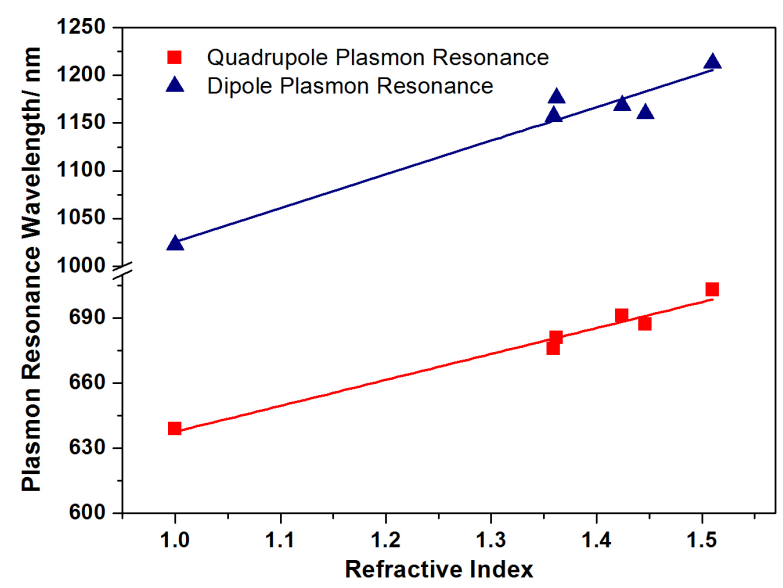

FIG. 10: Evolution of the experimental LSPP resonance wavelengths of the dipole (blue) and quadrupole (red) resonance for the NPs array $\mathrm{AR}_{3.6}^{*}$ with the refractive index of different surrounding solvents.

For the NPs array $\mathrm{AR}_{3.6}^{*}$, a very large sensitivity factors of $353 \mathrm{~nm} / \mathrm{RIU}$ and $120 \mathrm{~nm} / \mathrm{RIU}$ were measured for dipole and quadrupole resonance (Fig. 10).

We have demonstrated that triangular NPs arrays designed with the largest colloidal nanospheres (largest edge length) and smallest gold thickness are the most sensitive and the best candidate for the design of (bio)sensors in the near-infrared region. 


\section{Conclusions}

In this study, the optical properties of large triangular NPs prepared by nanosphere lithography have been investigated. We have shown that besides the dipole mode, multipolar plasmon modes are excited in these NPs arrays. The dipole and quadrupole modes, as identified by COMSOL simulations, red shifts as the edge length of the triangles increases for a given gold thickness. These resonances are well resolved allowing us to investigate them individually in the context of sensing applications. Additionally we find that increasing the edge length of the triangles the tips of the NPs became more sharper and in this way larger amplification of the near field are reached on the sharpest tips. As a consequence the NPs prepared with largest colloidal nanospheres are better candidates for sensing applications due to their high sensitivity factors. These results open the way to a new design of plasmonic structures as building blocks for sensing applications.

Acknowledgement: R.M and P.D acknowledge financial support from the EU-FEDER and Interreg IV, project PlasmoBio. B.K acknowledges financial support from Smart film grant 830039 (ECV12020020892F) in the framework of Convergence Project. R.M, P.D, B.K and acknowledge financial support of FRS-FNRS. H.S. and B.M acknowledge financial support by the IWT via SBO-project No. 060843 Polyspec, by the Interuniversity Attraction Poles program of Belspo Grant No. IAP P6-10 photonics@be and by COST action MP0702. R.V. acknowledges FRS-FNRS for a visiting professor grant. All authors warmly acknowledge Prof. Koen Clays (KU Leuven) for providing setup for optical measurements.

[1] M. A. Mahmoud and M. A. El-Sayed, J. Am. Chem. Soc., 2010, 132, 1270412710.

[2] J. N. Anker, W. P. Hall, O. Lyandres, N. C. Shah, J. Zhao and R. P. Van Duyne, Nat. Mater., $2008,7,442$.

[3] S. Enoch, R. Quidant, G. Badenes, Optics Express, 2004, 12, 3422.

[4] J. Z. Zhang, C. Noguez, Plasmonics, 2008, 3, 127.

[5] P. L. Stiles, J. A. Dieringer, N. C. Shah, R. P. Van Duyne, Ann. Rev. Anal. Chem., 2008, 1, 601.

[6] B. C. Galarreta, P. R. Norton, and F. L. Labarthet, Langmuir, 2011, 27 (4), 1494.

[7] M. Alschinger, M. Maniak, F. Stietz, T. Vartanyan, F. Träger, Appl. Phys. B, 2003, 76, 771. 
[8] F. Hubenthal, R. Morarescu, L. Englert, L. Haag, T. Baumert, F. Träger, Appl. Phys. Lett., 2009, 95, 063101.

[9] R. Morarescu, L. Englert, B. Kolaric, P. Damman, R. A. L. Vallée, T. Baumert, F. Hubenthal, F. Träger, J. Mater. Chem., 2011, 21, 4076.

[10] A. Abass, H. Shen, P. Bienstman, B. Maes, J. Appl. Phys., 2011, 109, 023111.

[11] H. Shen, P. Bienstman, B. Maes, J. Appl. Phys., 2009, 106(7), 073109.

[12] T. Ito, S. Okazaki, Nature, 2000, 406, 1027.

[13] H. Duan, J. Zhao, Y. Zhang, E. Xie, L. Hanhh,Nanotechnology, 2009, 20, 135306.

[14] N. Puttaraksa, S. Gorelick, T. Sajavaara, M. Laitinen, S. Singkarat, H. J. Whitlow, J. Vac. Sci. Technol. B, 2008, 26, 1732.

[15] C. L. Haynes, R. P. Van Duyne, J. Phys. Chem. B 2001, 105, 5599.

[16] T. R. Jensen, M. D. Malinsky, C. L. Haynes, R. P. Van Duyne, J. Phys. Chem. B, 2000, 104, 10549 .

[17] L. J. Sherry, R. Jin, C. A. Mirkin, G. C. Schatz, R. P. Van Duyne, Nano. Lett., 2006, 6, 2060.

[18] John C. Hulteen, R. P. Van Duyne, J. Vac. Sci. Technol. A, 1995, 13(3), 07342101.

[19] R. Micheletto, H. Fukuda, M. Ohtsu, Langmuir, 1995, 11, 3333.

[20] K. L. Kelly, E. Coronado, L. L. Zhao, G. C. Schatz, J. Phys. Chem. B, 2003, 107, 668.

[21] P. Yang, H. Portalès, M. P. Pileni, J. Phys. Chem. C, 2009, 113, 11597.

[22] R. Morarescu, D. B. Sánchez, N. Borg, T. A. Vartanyan, F. Träger, F. Hubenthal, Appl. Surf. Sci., 2009, 255, 9822 .

[23] K. L. Shuford, M. A. Ratner, G. C. Schatz, J. Chem. Phys., 2005, 123, 114713.

[24] A. J. Haes, S. Zou, G. C. Schatz, R. P. Van Duyne, J. Phys. Chem. B, 2004, 108, 109. 

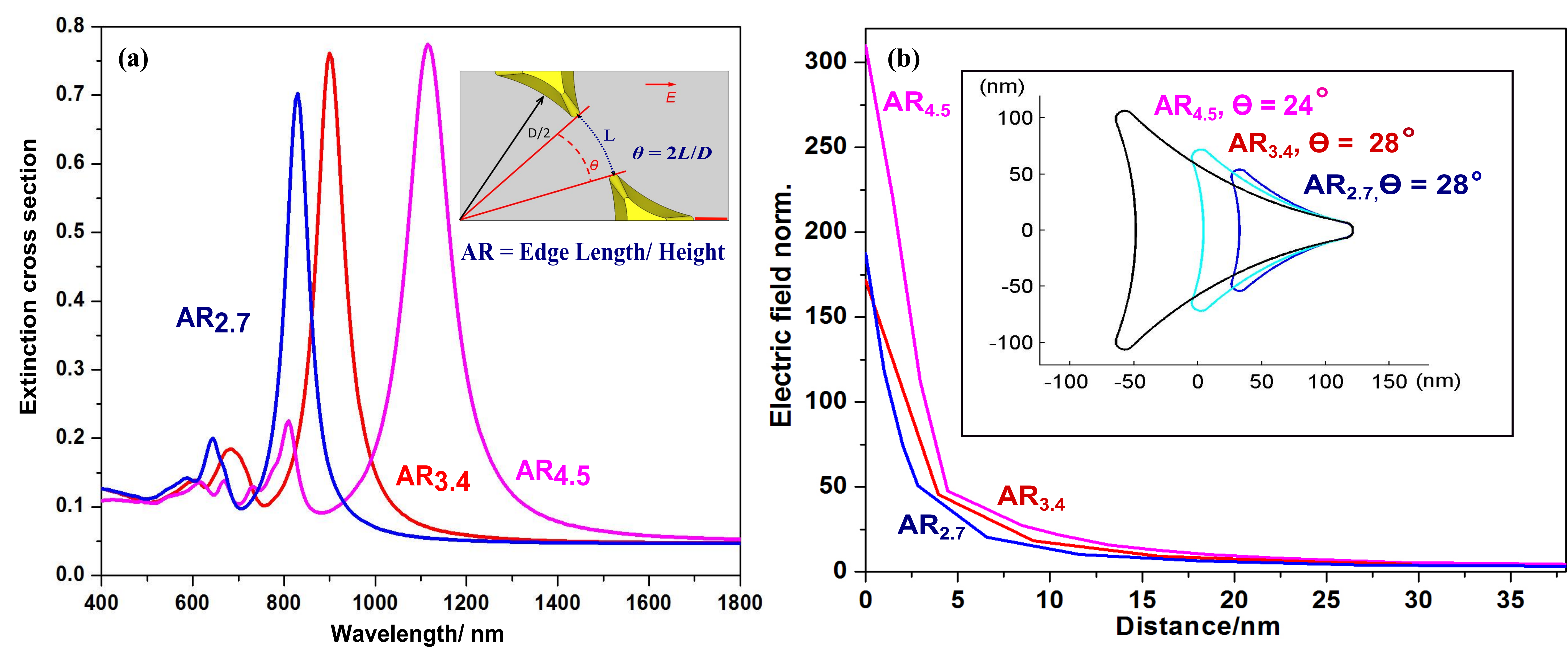


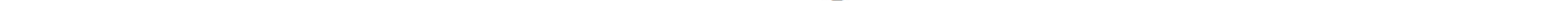



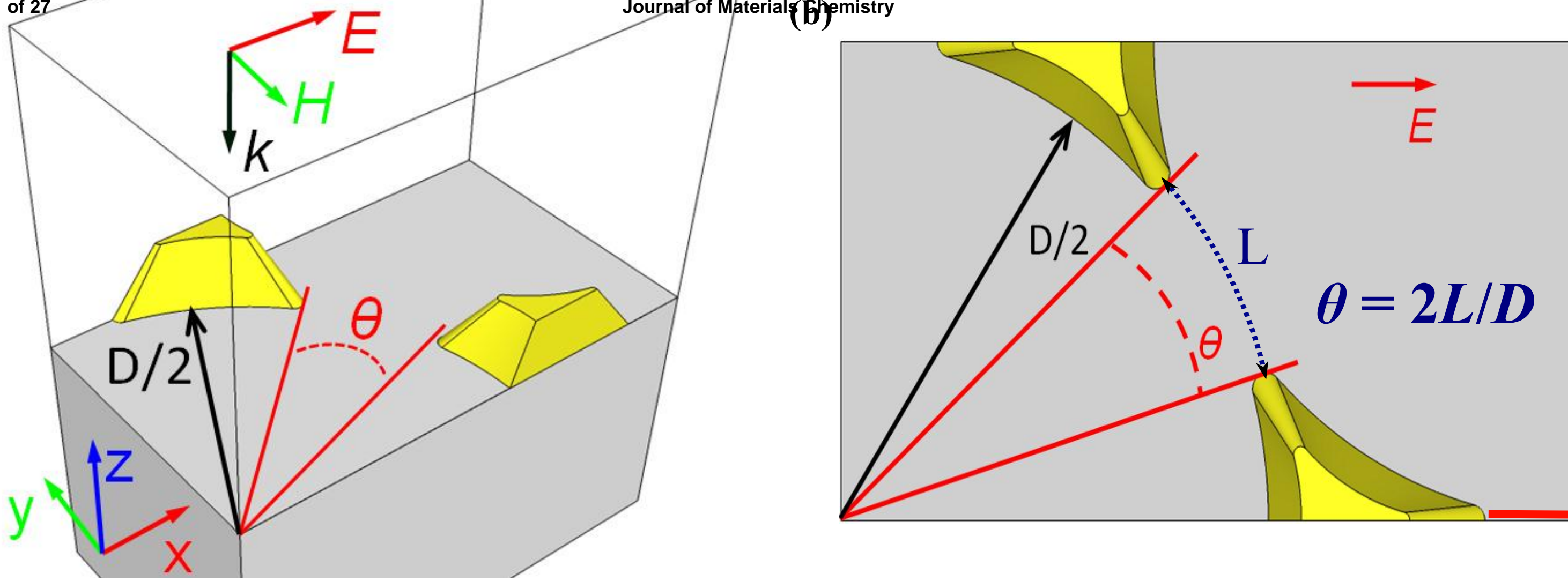


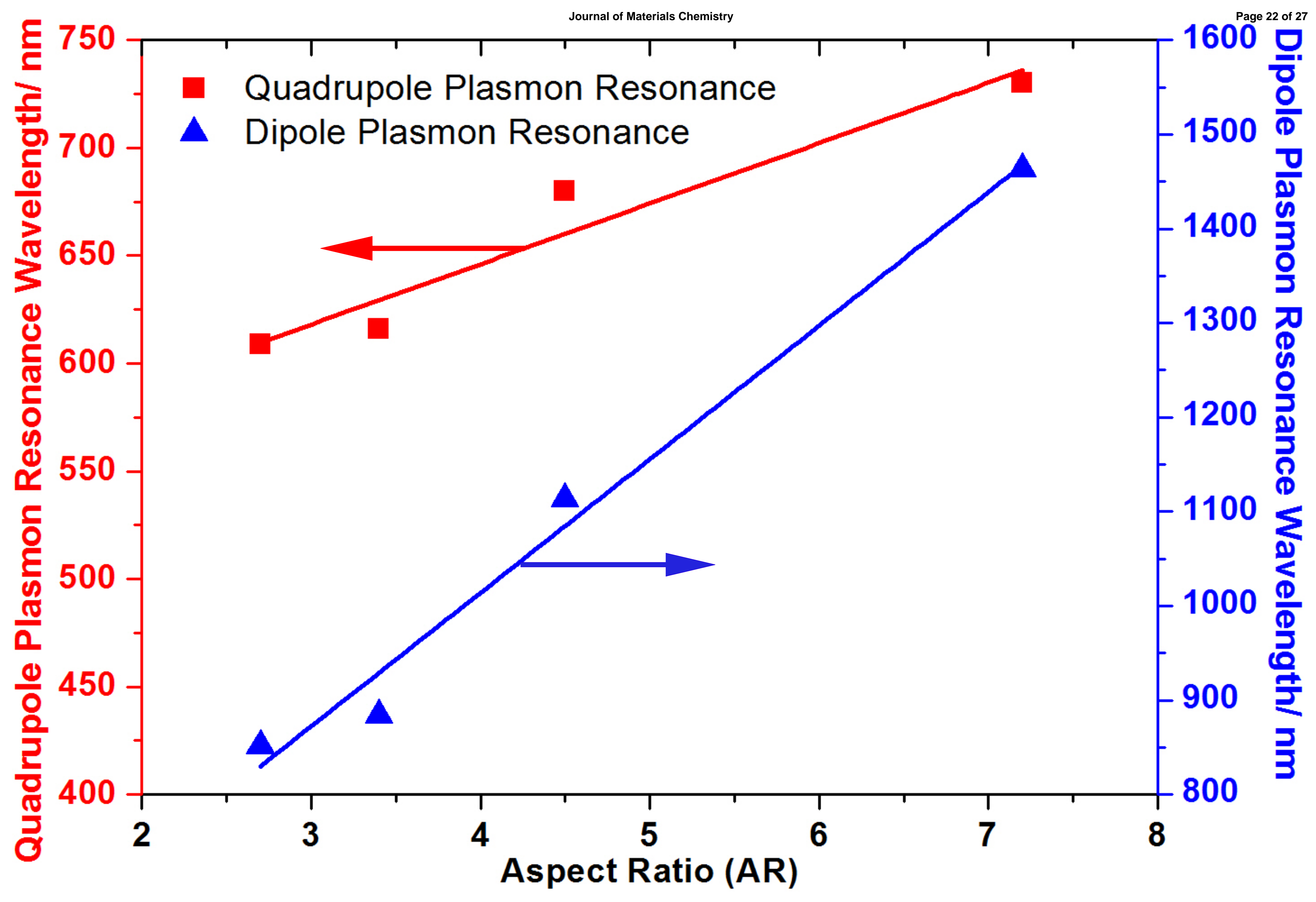




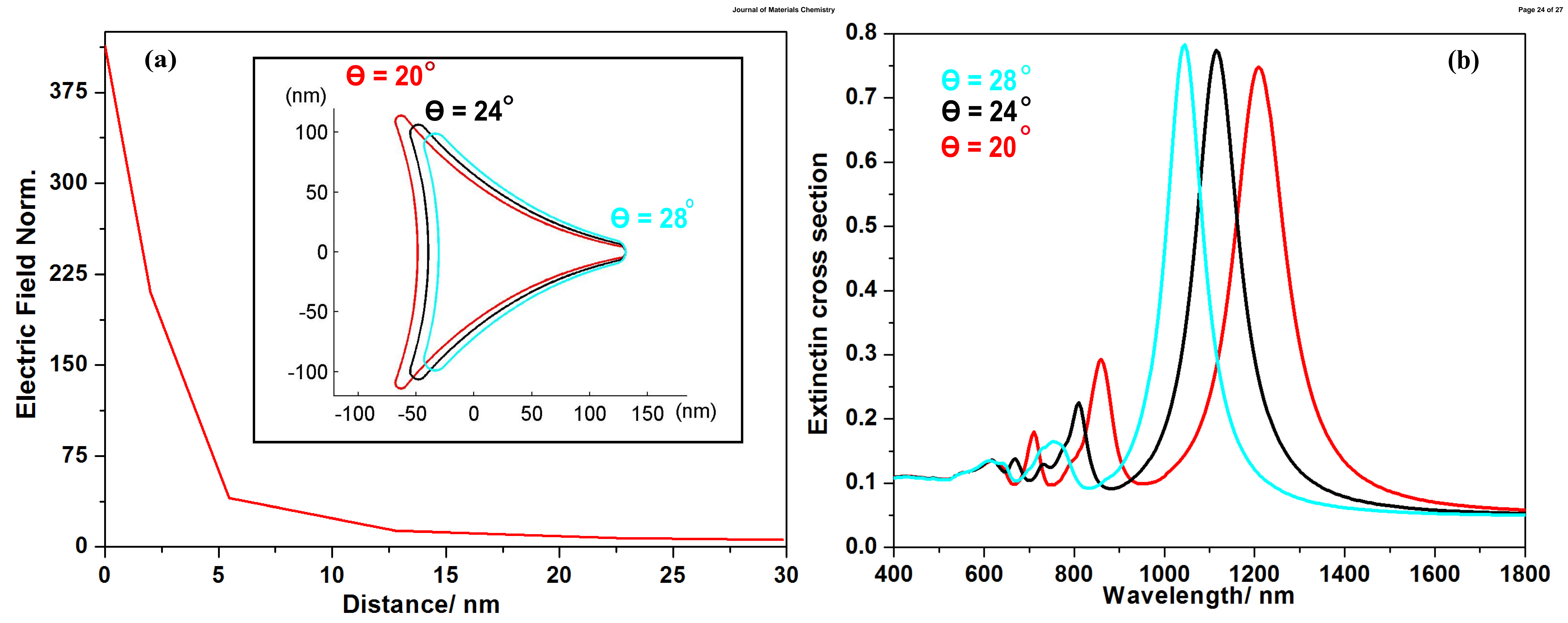



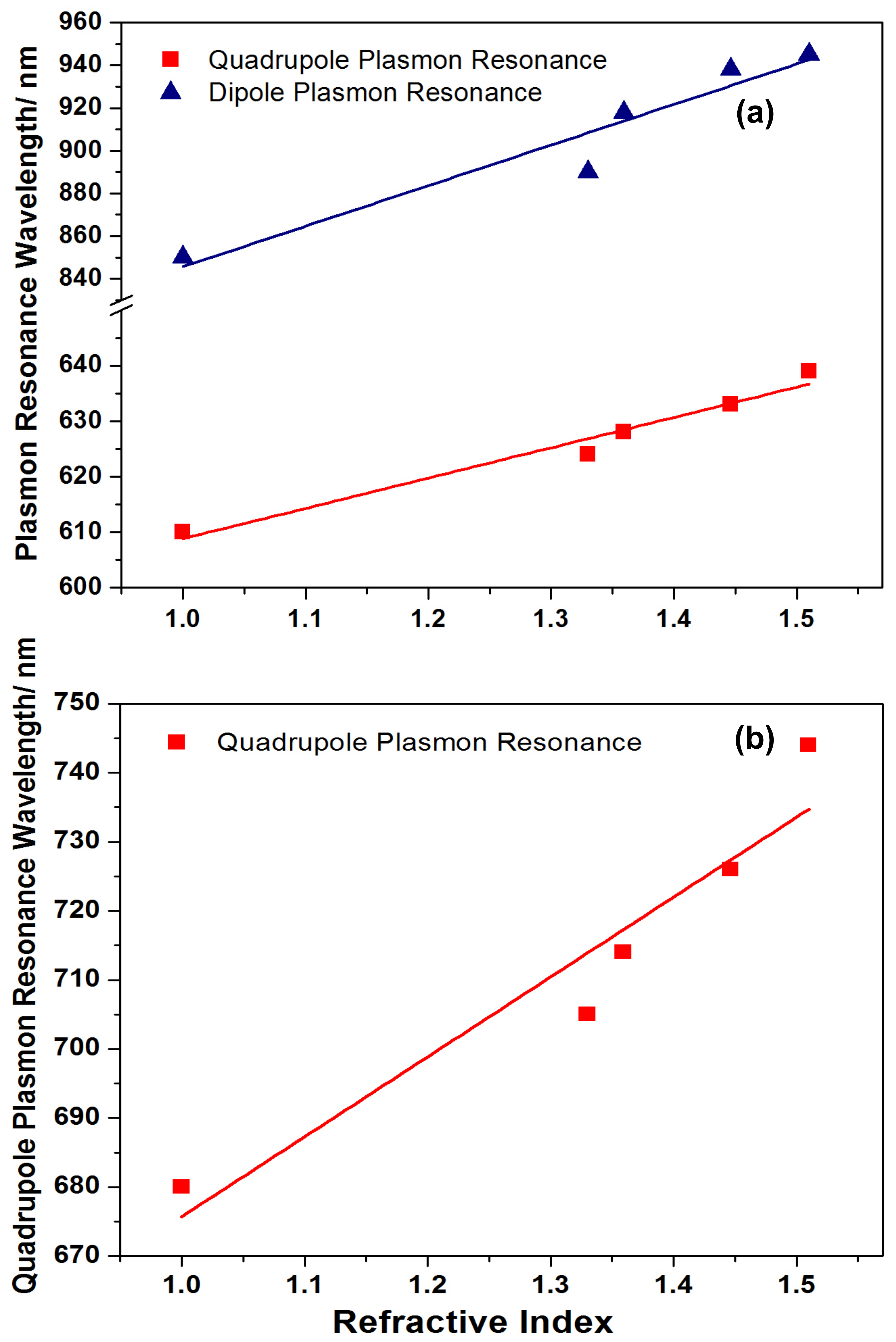


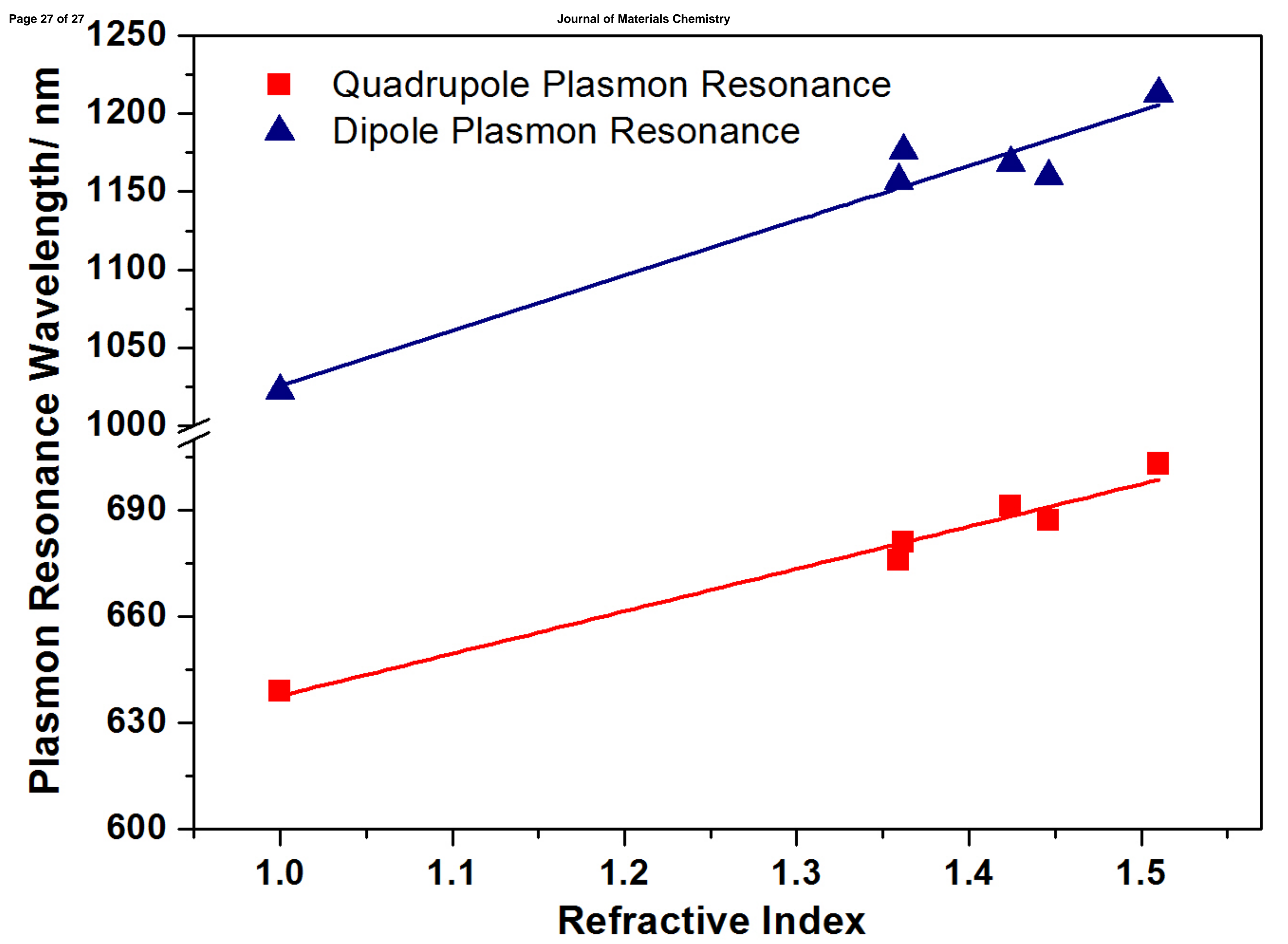

